\title{
ON CONVOLUTION TYPES IN COMMUTATIVE RINGS
}

\author{
Horst Brunotte \\ Received: 17 February 2014; Revised: 19 July 2014 \\ Communicated by Sarah Glaz \\ Dedicated to Professor Franz Halter-Koch on the occasion of his 70th birthday \\ Abstract. For a commutative associate unital ring $R$ the notion of an $R$ - \\ convolution type is introduced and applied to $R$-algebras. Some examples are \\ presented and relations to known concepts are discussed.
}

Mathematics Subject Classification (2010): 16S99, 13F99, 13F20, 13F25

Keywords: Convolution, convolution ring, convolution type

\section{Introduction}

Convolutions play important roles in many different areas of mathematics, e.g., functional analysis, algebra, logic, probability theory, group theory, number theory or measure theory. Concentrating on algebraic aspects the notion of a convolution ring is investigated and discussed by Fotino [5], Pierce [8], Rowen [10], Porst [9], Komatsu et al. [6], Aguiar and Mahajan [1], to mention a few.

Some years ago Veldsman [12] presented and studied a rather general convolution concept. Specifically, he investigated radical theoretic properties of rings originating from apparently widely different ring constructions and suggested a new grouping of seemingly disparate ring constructions. To this end he introduced the notion of convolution rings in a very general algebraic setting and showed that many important ring constructions can be covered under this umbrella. Furthermore, he investigated the influence of the so-called convolution type on the corresponding convolution ring. In a subsequent paper [13] he defined a more specialized convolution type, namely an arithmetic convolution type thereby providing a unified treatment of many rings which have been called arithmetic.

In this short note we further exploit the ideas of Veldsman. We consider a commutative associate unital ring $R$ and suggest the definition of an $R$-convolution type $\mathcal{T}$. At appropriate places the relation of this new notion to known concepts is commented. For a given $R$-algebra $A$ we study some ring properties of the set $C(A, \mathcal{T})$ of certain functions with values in $A$; in particular, some results on 
zero-divisors and units of $C(A, \mathcal{T})$ are established. Various definitions of particular convolution types which were introduced by Veldsman [13] are easily extended and used in our surroundings. Our considerations are illustrated by several examples of $R$-convolution types.

\section{Definition and examples of $R$-convolution types}

For the purpose of this note we introduce the following notion which in essence stems from work by Veldsman [13,12] and which was slightly modified in [3]. The reader is referred to [12] for a detailed discussion of the meaning and consequences of the properties listed below.

Throughout we let $R$ be an associative and commutative ring, and we assume that $R$ is unital, i.e., it has a multiplicative identity element which is different from the additive neutral element. In the definition given below it is convenient to regard $\rho$ as the convolution rule, $I$ as the index set and $T$ as the subset of trivial elements of $I$; the mapping $\tau$ is sometimes called the weighting kernel (e.g., see [5]).

Definition 1. Let $I$ be a non-empty set, $\mathcal{S}$ a subset ${ }^{1}$ of $\mathcal{P}(I)$ with $\mathcal{S} \neq\{I\}$ and $\rho: I \rightarrow \mathcal{P}(I \times I)$ and $\tau: I \times I \rightarrow R$ be mappings. The quadruple $\mathcal{T}=(I, \mathcal{S}, \rho, \tau)$ is called an $R$-convolution type if it enjoys the following properties.

(C1) For all $S_{1}, S_{2} \in \mathcal{S}$ there is an $S \in \mathcal{S}$ such that $S \subseteq S_{1} \cap S_{2}$.

(C2) The intersection of all $S \in \mathcal{S}$ is empty.

(C3) Given $S_{1}, S_{2} \in \mathcal{S}$ there exists an $S \in \mathcal{S}$ with the following property: For all $s \in S$ the relation $(i, j) \in \rho(s)$ implies $i \in S_{1}$ or $j \in S_{2}$.

(C4) For all $i \in I$ and $S_{1}, S_{2} \in \mathcal{S}$ we have

$$
\operatorname{card}\left\{(r, s) \in \rho(i): r \in I \backslash S_{1}, s \in I \backslash S_{2}\right\}<\infty .
$$

(C5) For all $i \in I,(r, s) \in \rho(i)$ and $(p, q) \in \rho(r)$ there exists a unique $u \in I$ such that $(p, u) \in \rho(i),(q, s) \in \rho(u)$ and

$$
\tau(r, s) \tau(p, q)=\tau(p, u) \tau(q, s) .
$$

(C6) For all $i \in I,(r, s) \in \rho(i)$ and $(p, q) \in \rho(s)$ there exists a unique $v \in I$ such that $(v, q) \in \rho(i),(r, p) \in \rho(v)$ and

$$
\tau(r, s) \tau(p, q)=\tau(v, q) \tau(r, p) .
$$

(C7) For all $i \in I,(p, q) \in \rho(i)$ we have $q=i$ provided $p$ belongs to the set

$$
T:=\{t \in I:(t, t) \in \rho(t) \text { and } \tau(t, j)=\tau(j, t)=1 \text { for all } j \in I\}
$$

${ }^{1} \mathcal{P}(X)$ denotes the set of all subsets of the set $X$. 
where 1 denotes the multiplicative identity element of $R$.

(C8) For every $i \in I$ there are uniquely determined $s, t \in T$ such that

$$
(t, i) \in \rho(i) \quad \text { and } \quad(i, s) \in \rho(i)
$$

(C9) There is some $S \in \mathcal{S}$ such that $T \subseteq I \backslash S$.

Let us briefly comment on this definition and its relation to known concepts.

Remark 2. (i) In our definition above the values of $\tau$ need not necessarily be rational integers. Therefore, apart from property (C2) our notion is slightly more general than the ones used in $[13,3]$.

(ii) Note that $T, \mathcal{S} \neq \emptyset$ in view of (C8) and (C9), respectively.

(iii) If $\emptyset \in \mathcal{S}$ then (C1), (C2), (C3) and (C9) are trivially satisfied.

(iv) As can be seen in the examples below it fairly commonly occurs that $\tau$ is symmetric, i.e., we have $\tau(i, j)=\tau(j, i)$ for all $i, j \in I$.

(v) If $T=I$ then $\tau=1$, i.e., we have $\tau(i, j)=1$ for all $i, j \in I$.

(vi) Let $\tau=1$. Then we directly find $T=\{t \in I:(t, t) \in \rho(t)\}$.

(vii) If $\rho$ is symmetric, i.e., $\rho$ satisfies the property

$$
(r, s) \in \rho(i) \Longleftrightarrow(s, r) \in \rho(i) \quad(i, r, s \in I),
$$

then only one of the conditions (C5) or (C6) needs to be verified (cf. [12, Section 1]).

(viii) The occurrence of property (C2) is rather common (cf. Example 4 below).

(ix) Property (C4) is trivially satisfied if card $\rho(i)<\infty$.

(x) Let us suppose that $I$ is contained in the set $\mathbb{N}$ of positive rational integers and that

$$
(i, j) \in \rho(k) \Longleftrightarrow i j=k \quad(i, j, k \in I)
$$

Under this condition we have

$$
\tau(i, j) \tau(i j, k)=\tau(i, j k) \tau(j, k) \quad(i, j, k \in I)
$$

by (C5). Fotino [5, Section 3] observed that in algebraic topology solutions of equation (2) are viewed as cocycles; moreover, if $R$ is an integral domain then (symmetric) functions of the shape

$$
\tau(i, j)=\frac{\alpha(i j)}{\alpha(i) \alpha(j)} \quad(i, j \in I)
$$

with a non-vanishing function $\alpha: I \rightarrow R$ (i.e., $\alpha(i) \neq 0$ for all $i \in I$ ) are viewed as coboundaries. 
In the remainder of this note we let $\mathcal{T}=(I, \mathcal{S}, \rho, \tau)$ be an $R$-convolution type and $A$ be a unital $R$-algebra (see [7]). Following [12] we set

$C(A, \mathcal{T})=\{f: I \rightarrow A:$ there exists some $S \in \mathcal{S}$ such that $f(s)=0$ for all $s \in S\}$ and define two operations on $C(A, \mathcal{T})$ : For $f, g \in C(A, \mathcal{T})$ and $i \in I$ we set

$$
(f+g)(i)=f(i)+g(i)
$$

and

$$
(f \bullet g)(i)=\sum_{(u, v) \in \rho(i)} \tau(u, v) f(u) g(v) .
$$

Now we show some algebraic properties of $C(A, \mathcal{T})$.

Proposition 3. (i) $(C(A, \mathcal{T}),+, \bullet)$ is a unital ring with multiplicative identity element $\iota_{1_{A}}$ where $1_{A}$ denotes the multiplicative identity in $A$ and the mapping $\iota_{a}: I \rightarrow A$ is defined by

$$
\iota_{a}(i)= \begin{cases}a & (i \in T) \\ 0 & (i \notin T)\end{cases}
$$

for $a \in A$.

(ii) The mapping $\iota: A \rightarrow C(A, \mathcal{T})$ given by $\iota(a)=\iota_{a}$ defines a ring monomorphism.

(iii) For $r \in R$ and $f, g \in C(A, \mathcal{T})$ we have

$$
r(f+g)=r f+r g \quad \text { and } \quad r(f \bullet g)=(r f) \bullet g .
$$

(iv) Let $A$ be commutative and assume that $\tau$ is symmetric and that $\rho$ enjoys property (1). Then $C(A, \mathcal{T})$ is commutative.

Proof. Note that by $(\mathrm{C} 9)$ the mappings $\iota_{a}$ belong to $C(A, \mathcal{T})$.

(i), (ii) Clear by $[12$, Section 1$]$.

(iii), (iv) This can immediately be checked by the definitions.

Based on these observations we regard $A$ as a subring of $C(A, \mathcal{T})$ and identify $a$ and $\iota_{a}$ for all $a \in A$. Clearly, if $I$ possesses only one element then $\mathcal{S}=\{\emptyset\}, \tau=1$, and $C(A, \mathcal{T})$ and $A$ coincide.

Some examples which are inspired by known concepts conclude this section.

Example 4. (i) Let $\mathcal{T}$ be a discrete convolution type in the sense of [3, Definition 1]. If $\mathcal{T}$ fulfills (C2) then it is a $\mathbb{Z}$-convolution type; here we denote by $\mathbb{Z}$ the set of rational integers. Observe that in all examples listed in [3, Example 4] property (C2) is fulfilled. In particular, replacing $\mathbb{Z}$ by $R$ 
all examples mentioned in [3, Example 4 (i)] can be seen as $R$-convolution types.

(ii) Adopting the notion coined in [13] we let $(X, \sigma)$ be an arithmetic convolution type such that $X$ contains a non-empty subset $T$ defined in $[13$, Section 2]. As observed in [3, Remark 2] the quadruple $(X,\{\emptyset\}, \sigma, 1)$ is a discrete convolution type, thus a $\mathbb{Z}$-convolution type by $(i)$.

(iii) [12, Example 2.6] Fix $m \in \mathbb{N}$ and define

$$
I:=\{(i, j): i, j \in\{1, \ldots, m\}\}
$$

Then $\mathcal{T}:=(I,\{\emptyset\}, \rho, 1)$ is an $R$-convolution type where we define

$$
\rho(i, j)=\{((i, k),(k, j)): k \in\{1, \ldots, m\}\} .
$$

Moreover, in this case $C(R, \mathcal{T})$ is isomorphic to the full $m \times m$ matrix ring over $R$.

(iv) [12, Example 2.10] Let $G$ be a multiplicatively written semigroup with identity and at least two elements, $\mathcal{S}:=\{S \subseteq G: G \backslash S$ finite $\}$ and

$$
\rho(g):=\{(s, t): s, t \in G, s t=g\} .
$$

Then $(G, \mathcal{S}, \rho, 1)$ is an $R$-convolution type.

(v) [12, Example 2.11] Let $G$ be a multiplicatively written finite group. Suppose that $G$ possesses an element $u$ such that $u^{2}$ equals the identity and such that $G$ contains a subset $S$ with the property

$$
s \in S \Longleftrightarrow u s \notin S \text {. }
$$

Fix $d \in\{-1,1\} \subseteq R$. For $g, h \in G$ define

$$
\rho(g):=\{(s, t): s, t \in G, s t=g \text { or } s t=u g\}
$$

and

$$
\tau(g, h):=\left\{\begin{aligned}
1, & \text { if } g h \in S, \\
d, & \text { if } g h \notin S .
\end{aligned}\right.
$$

Then $(G,\{\emptyset\}, \rho, \tau)$ is an $R$-convolution type.

(vi) The example given by Bhattacharjee and Saikia [2, Example 5.1] under the name of an $S_{B}$-product can be modified. Let $I$ be a multiplicatively closed subset of the positive integers which contains 1 , and set $\mathcal{S}:=\{I, \emptyset\}$. Further, define the convolution rule by

$$
\rho(i):=\{(1, i),(i, 1)\} \quad(i \in I),
$$


and let $\tau: I \times I \rightarrow R$ be a symmetric map such that $\tau(1, i)=1$ for all $i \in I$. Obviously, $\rho$ is symmetric, and it can straightforwardly be checked that $(I, \mathcal{S}, \rho, \tau)$ is an $R$-convolution type; clearly, we have $T=\{1\}$.

Observe that in the definition of $\rho(i)$ we cannot take the identical set as in [2, Example 5.1]. The reason lies in the requirement of the uniqueness of the element $u$ in property (C5); this uniqueness is used in the proof of the associativity of the ring $C(A, \mathcal{T})$ in Proposition 3. More precisely, our proof exploits a bijective relation between summands of certain sums while in [2, Theorem 2.2] associativity is achieved by a condition on these sums themselves.

\section{Zero-divisors and units in algebras with an $R$-convolution type}

In this section we continue to keep our conventions; in particular, we let $\mathcal{T}$ be an $R$-convolution type.

Let us first construct some useful elements in the ring $C(A, \mathcal{T})$.

Lemma 5. Let $M$ be a finite nonvoid subset of $I$ and $f: I \rightarrow A$ be a mapping such that $f(i)=0$ for all $i \in I \backslash M$. Then $f$ belongs to $C(A, \mathcal{T})$.

Proof. We use induction on the number of elements of $M$ in order to show that there exists some $S \in \mathcal{S}$ such that $S \subseteq I \backslash M$. Observe that then $f(s)=0$ for all $s \in S$, thus we have $f \in C(A, \mathcal{T})$.

If $M=\{i\}$ then by (C2) there is some $S \in \mathcal{S}$ such that $i \notin S$, and we clearly have $S \subseteq I \backslash M$. Now, let card $M>1$ and pick non-empty disjoint subsets $M_{1}, M_{2}$ of $M$ such that $M=M_{1} \cup M_{2}$. In view of $0<\operatorname{card} M_{1}$, $\operatorname{card} M_{2}<\operatorname{card} M$ and our induction hypothesis there exist $S_{1}, S_{2} \in \mathcal{S}$ such that $S_{1} \subseteq I \backslash M_{1}$ and $S_{2} \subseteq I \backslash M_{2}$. By (C1) there is an $S \in \mathcal{S}$ such that $S \subseteq S_{1} \cap S_{2}$. Certainly, we have $S \subseteq I \backslash M$.

An application of this lemma shows that $C(A, \mathcal{T})$ can have zero-divisors irrespective of whether $A$ has or has not.

Proposition 6. $C(A, \mathcal{T})$ has non-zero zero-divisors if one of the following two conditions is satisfied.

(i) There exist $p, q \in I$ such that $(p, q) \notin \rho(i)$ for every $i \in I$.

(ii) There exists an element $p \in I \backslash T$ such that $(p, p) \in \rho(p)$ and $(p, p) \notin \rho(i)$ for every $i \in I \backslash\{p\}$.

Proof. (i) The proof of [13, Proposition 2] can be copied (cf. [3, Propostion 5]).

(ii) We adapt the proof of [13, Proposition 3]. By (C8) there is a $t \in T$ such that 
$(t, p) \in \rho(p)$. Pick $a \in A \backslash\{0\}$ and define

$$
f(i)=\left\{\begin{array}{ll}
-a, & \text { if } i=p, \\
\tau(p, p) a, & \text { if } i=t, \\
0, & \text { otherwise }
\end{array} \quad \text { and } \quad g(i)=\left\{\begin{array}{ll}
-a, & \text { if } i=p, \\
0, & \text { otherwise }
\end{array} \quad(i \in I)\right.\right.
$$

Then $f, g \in C(A, \mathcal{T})$ by Lemma 5 . Further we have $f, g \neq 0$, and we find $f \bullet g=0$ since

$$
\begin{aligned}
(f \bullet g)(p) & =\sum_{(u, v) \in \rho(p)} \tau(u, v) f(u) g(v)=\sum_{(u, p) \in \rho(p)} \tau(u, p) f(u)(-a) \\
& =\tau(p, p) f(p)(-a)+\tau(t, p) f(t)(-a)=\tau(p, p)(-a)(-a)+\tau(p, p) a(-a) \\
& =\tau(p, p)(-a+a)(-a)=0
\end{aligned}
$$

and for $i \neq p$

$$
(f \bullet g)(i)=\sum_{(u, p) \in \rho(i)} \tau(u, p) f(u)(-a)=0
$$

because $(p, p),(t, p) \notin \rho(i)$.

From now on we assume that there is a linear order on $I$ denoted by $\leq$. For the convenience of the reader we recall the following notions which were coined by Veldsman [13, Section 3] for the important case $I \subseteq \mathbb{Z}$. Similarly as in [13, Section $3]$ and [3, Section 3] we aim at excluding zero-divisors in $C(A, \mathcal{T})$ which do not belong to $A$.

Definition 7. We say that $\mathcal{T}$

- fulfills the lower bound requirement if $I \backslash T$ has a lower bound in $I$ provided that $I \neq T$.

- has the complementary ordering property if for all $i \in I$ and all $(r, s),(u, v) \in$ $\rho(i)$ we have

$$
r \leq u \Longleftrightarrow s \geq v
$$

- is well-behaved if it satisfies the following three properties.

(i) $\mathcal{T}$ fulfills the lower bound requirement.

(ii) $\mathcal{T}$ has the complementary ordering property.

(iii) For all $i, j \in I$ there exists some $k \in I$ such that $(i, j) \in \rho(k)$.

Remark 8. [13, Section 3]

(i) Suppose that $\mathcal{T}$ has the complementary ordering property.

(a) Let $i \in I$ and $(r, s),(u, v) \in \rho(i)$ such that $r<u$. Then we have $s>v$. 
(b) The second condition mentioned in Proposition 6 is not satisfied. More precisely, there does not exist an element $p \in I \backslash T$ such that

$$
(p, p) \in \rho(p) \backslash \bigcup_{i \in I} \rho(i) .
$$

(ii) Let $\mathcal{T}$ be well-behaved. Then card $T=1$, i.e., I possesses exactly one trivial element: By Remark 2 (ii) we have card $T \geq 1$. Suppose that there exist $p, q \in T$ such that $q<p$, and pick $k \in I$ such that $(q, p) \in \rho(k)$. Property (C7) yields $k=p$, hence $(q, p) \in \rho(p)$. Using property (C8) we find $s \in T$ such that $(p, s) \in \rho(p)$. But this yields $s=p$ by $(C r)$, while the complementary ordering property requires $p>s$ : Contradiction.

Moreover, none of the two conditions mentioned in Proposition 6 is satisfied.

As explained in [13] a well-behaved convolution type imposes a strong algebraic structure on the set $I$. We formulate the result [13, Proposition 7] as follows.

Proposition 9. Let $\rho$ be symmetric and $\mathcal{T}$ be a well-behaved $R$-convolution type. For any $i, j \in I$ there is a unique $k \in I$ such that $(i, j) \in \rho(k)$; in this case we write $k=i * j$. Moreover, $(I, *)$ is a commutative cancellative semigroup with the unique trivial element as identity. For all $i, j, k \in I$ the relation $i<j$ implies $i * k<j * k$. If card $I>1$ then $I$ is infinite.

Proof. The proofs of [13, Lemma 6 and Proposition 7] hold under our prerequisites. As an example we verify $[13$, Lemma $6(\mathrm{v})]$, i.e., we show that if

$$
(r, s) \in \rho(i)
$$

then any two elements of $i, r, s$ determine the third uniquely. Indeed, the assumption $(u, s) \in \rho(i)$ yields $r=u$ by the complementary ordering property. Now, let

$$
(r, s) \in \rho(j)
$$

with some $j \in I$. Since $\mathcal{T}$ is a well-behaved there exists some $k \in I$ such that

$$
(i, j) \in \rho(k)
$$

In view of (5) and (3) property (C5) of Definition 1 yields some $v \in I$ such that $(r, v) \in \rho(k)$ and $(s, j) \in \rho(v)$. Analogously, $(j, i) \in \rho(k)$ and (4) imply the existence of some $u \in I$ such that $(r, u) \in \rho(k)$ and $(s, i) \in \rho(u)$. Thus we have $(r, v) \in \rho(k)$ and $(r, u) \in \rho(k)$. By what we have just seen above we conclude $u=v$. But then we have $j=i$ because we know $(s, j) \in \rho(u)$ and $(s, i) \in \rho(u)$. 
The criterion on the existence of zero-divisors in [13, Proposition 4] can be extended to our settings here and we record the result as follows.

Theorem 10. Suppose that every non-empty subset of I which is bounded from below has a minimal element. Further, let $\mathcal{T}$ be a well-behaved $R$-convolution type such that for all $r, s \in I$ the element $\tau(r, s)$ is not a zero-divisor in $A$. Then $C(A, \mathcal{T})$ has zero-divisors if and only if $A$ has zero-divisors.

Proof. We first claim that $I$ has a minimal element. Indeed, by Remark 8 there is some $t \in I$ such that $T=\{t\}$. Thus our assertion is trivial if $I=T$, and we let $I \neq T$. By the lower bound requirement there exists $k \in I$ such that $k$ is a lower bound for $I \backslash T$. Then $\min \{t, k\}$ is a minimal element of $I$.

Now, the proof of [13, Proposition 4] can be adapted.

This theorem and Proposition 3 (iii) immediately yield the following result.

Corollary 11. Suppose that every non-empty subset of I which is bounded from below has a minimal element. Let $R$ be an integral domain and $\mathcal{T}$ be a well-behaved $R$-convolution type such that $\tau(r, s) \neq 0$ for all $r, s \in I$. Then $C(R, \mathcal{T})$ is an integral domain.

Let us now turn to units in $C(R, \mathcal{T})$. Analogously as in [13, Section 4] we present conditions which guarantee that $C(R, \mathcal{T})$ has more units than $R$.

Theorem 12. Let $I$ be contained in $\mathbb{N}$. Further assume that $\mathcal{T}$ is an $R$-convolution type which satisfies the lower bound requirement and the following two conditions.

(i) For all $t \in T$ we have $\rho(t)=\{(t, t)\}$.

(ii) Let $i, j, k \in I$ and $(i, j) \in \rho(k)$. If $i \notin T$ then $j<k$.

Then $f \in C(R, \mathcal{T})$ is a unit if and only if $f(t)$ is a unit in $R$ for all $t \in T$.

Proof. The proof of [3, Theorem 12] which is adapted from the proof of [13, Proposition 5] can be copied.

Specializing to well-behaved $R$-convolution types yields the following characterization of units.

Corollary 13. Let $I \subseteq \mathbb{N}, \rho$ be symmetric and $\mathcal{T}$ be a well-behaved $R$-convolution type. Then $f \in C(R, \mathcal{T})$ is a unit if and only if $f(t)$ is a unit in $R$ for all $t \in T$.

Proof. In the proof of Proposition 9 we already mentioned that [13, Lemma 6] holds under our prerequisites. Therefore the assertion follows from the Theorem. 
Let us briefly return to an example given above.

Example 14. Under the additional condition card $I>1$ we extend our remarks on the $R$-convolution type $\mathcal{T}:=(I, \mathcal{S}, \rho, \tau)$ described in Example 4 (vi). By assumption $I$ contains some $i>1$, and we clearly have $(i, i) \notin \rho(k)$ for all $k \in I$, hence $\mathcal{T}$ is not well-behaved. By Proposition 6 the ring $C(A, \mathcal{T})$ has non-zero zero divisors; in fact, the proof of this proposition allows an easy construction of some zerodivisors. Further, we observe that $\mathcal{T}$ fulfills the lower bound requirement and has the complementary ordering property. In particular, if $R$ is a field then Theorem 12 yields that $f \in C(R, \mathcal{T})$ is a unit if and only if $f(1) \neq 0$.

Komatsu et al. [6, Section 3] defined a so-called $Q_{\alpha}$-convolution which may be extended in the following way.

Theorem 15. Let I be a multiplicatively closed subset of the positive integers which contains 1. Define

$$
\rho(i)=\{(r, s) \in I \times I: r s=i\} \quad(i \in I) .
$$

Let $F$ be a field and $\alpha: I \rightarrow F$ and $\beta: I \times I \rightarrow F$ be non-vanishing maps. If $\beta$ is bi-multiplicative, i.e.,

$$
\beta(i j, k)=\beta(i, k) \beta(j, k) \text { and } \beta(i, j k)=\beta(i, j) \beta(i, k) \quad(i, j, k \in I)
$$

then $\mathcal{T}:=(I,\{\emptyset\}, \rho, \tau)$ is an $F$-convolution type where $\tau: I \times I \rightarrow F$ is given by

$$
\tau(i, j):=\frac{\alpha(i j)}{\alpha(i) \alpha(j)} \cdot \beta(i, j) \quad(i, j \in I)
$$

The units of $C(F, \mathcal{T})$ are precisely the maps $f: I \rightarrow F$ such that $f(1) \neq 0$. Moreover, if $\beta=1$ then $C(F, \mathcal{T})$ is commutative.

Proof. In view of Remark 2 (iii), (vii) the verification of properties (C1), .., (C9) in Definition 1 can straightforwardly be performed. For instance, in order to check (C5) we let $i \in I,(r, s) \in \rho(i)$ and $(p, q) \in \rho(r)$. Then $r s=i$ and $p q=r$. Therefore, $u:=q s \in I$ since $I$ is closed under multiplication. Clearly, we have $(p, u) \in \rho(i),(q, s) \in \rho(u)$, and $u$ is uniquely determined since for $u^{\prime} \in I$ such that 
$(q, s) \in \rho\left(u^{\prime}\right)$ we have $u^{\prime}=q s=u$. Further, we have

$$
\begin{aligned}
\tau(r, s) \tau(p, q) & =\frac{\alpha(i)}{\alpha(r) \alpha(s)} \cdot \frac{\alpha(r)}{\alpha(p) \alpha(q)} \cdot \beta(p q, s) \cdot \beta(p, q) \\
& =\frac{\alpha(i)}{\alpha(s)} \cdot \frac{1}{\alpha(p) \alpha(q)} \cdot \beta(p, s) \cdot \beta(q, s) \cdot \beta(p, q) \\
& =\frac{\alpha(p u)}{\alpha(p) \alpha(u)} \cdot \frac{\alpha(u)}{\alpha(s) \alpha(q)} \cdot \beta(p, q) \cdot \beta(p, s) \cdot \beta(q, s) \\
& =\frac{\alpha(p u)}{\alpha(p) \alpha(u)} \cdot \frac{\alpha(q s)}{\alpha(q) \alpha(s)} \cdot \beta(p, q s) \cdot \beta(q, s) \\
& =\tau(p, u) \tau(q, s) .
\end{aligned}
$$

Obviously, $\mathcal{T}$ is well-behaved. We observe that $T=\{1\}$, and then the characterization of the units of $C(F, \mathcal{T})$ follows from Theorem 12 .

Finally, if $\beta=1$ then $\tau$ is symmetric, and we infer from Proposition 3 that $C(F, \mathcal{T})$ is commutative.

Finally we show that the so-called generalized convolution ring of arithmetic functions studied by Shapiro [11], Fotino [5] and Ferrero [4] can be seen as a convolution type in our settings here.

Corollary 16. Let $\tau: \mathbb{N} \times \mathbb{N} \rightarrow \mathbb{C}$ be a non-vanishing map such that

$$
\tau(i, j) \tau(i j, k)=\tau(i, j k) \tau(j, k) \quad(i, j, k \in \mathbb{N}) .
$$

Then $\mathcal{T}:=(I,\{\emptyset\}, \rho, \tau)$ is a $\mathbb{C}$-convolution type where we set

$$
\rho(i)=\{(r, s) \in \mathbb{N} \times \mathbb{N}: r s=i\} \quad(i \in \mathbb{N}) .
$$

The units of $C(\mathbb{C}, \mathcal{T})$ are precisely the maps $f: \mathbb{N} \rightarrow \mathbb{C}$ such that $f(1) \neq 0$. Moreover, if $\tau$ is symmetric then $C(\mathbb{C}, \mathcal{T})$ is commutative.

Proof. We infer from [5, Theorem 3.1] that there exist a non-vanishing map $\alpha$ : $\mathbb{N} \rightarrow \mathbb{C}$ and a bi-multiplicative map $\beta: \mathbb{N} \times \mathbb{N} \rightarrow \mathbb{C}$ such that

$$
\tau(i, j)=\frac{\alpha(i j)}{\alpha(i) \alpha(j)} \cdot \beta(i, j) \quad(i, j \in \mathbb{N}) ;
$$

furthermore, if $\tau$ is symmetric then $\beta=1$. Now, our assertion follows from Theorem 15 .

Acknowledgement. The author is very grateful to an unknown referee for critical comments thanks to which the first draft of this paper was considerably improved. 


\title{
References
}

[1] M. Aguiar and S. Mahajan, Hopf monoids in the category of species, in Hopf algebras and tensor categories, Contemp. Math., 585, Amer. Math. Soc., Providence, RI, 2013, 17-124.

[2] D. Bhattacharjee and P. K. Saikia, On some new class of arithmetic convolutions involving arbitrary sets of integers, Rom. J. Math. Comput. Sci., 2 (2012), 23-35.

[3] H. Brunotte, Discrete convolution rings, Rom. J. Math. Comput. Sci., 3 (2013), $155-159$.

[4] M. Ferrero, On generalized convolution rings of arithmetic functions, Tsukuba J. Math., 4 (1980), 161-176.

[5] I. P. Fotino, Generalized convolution ring of arithmetic functions, Pacific J. Math., 61 (1975), 103-116.

[6] T. Komatsu, S. Srisopha, P. Ruengsinsub and V. Laohakosol, Results about dependence and convolution. Conference proceedings: Analytic Number Theory - Related Multiple Aspects of Arithmetic Functions, held at the Research Institute for Mathematical Sciences, October 31 to November 2, 2009, http://www.kurims.kyoto-u.ac.jp/kyodo/kokyuroku/contents/pdf/180607.pdf, 2012.

[7] S. Lang, Algebra, Graduate Texts in Mathematics, 211, Springer-Verlag, New York, Third ed., 2002.

[8] R. S. Pierce, Associative Algebras, Graduate Texts in Mathematics, 88. Studies in the History of Modern Science, 9. Springer-Verlag, New York-Berlin, 1982.

[9] H.-E. Porst, Dual adjunctions between algebras and coalgebras, Arab. J. Sci. Eng. Sect. C Theme Issues, 33 (2008), 407-411.

[10] L. H. Rowen, Ring Theory, Academic Press Inc., Boston, MA, student ed., 1991.

[11] H. N. Shapiro, On the convolution ring of arithmetic functions, Comm. Pure Appl. Math., 25 (1972), 287-336.

[12] S. Veldsman, Convolution rings, Algebra Colloq., 13 (2006), 211-238.

[13] S. Veldsman Arithmetic convolution rings, Int. J. Algebra, 5 (2011), 771-791.

\author{
Horst Brunotte \\ Haus-Endt-Straße 88 \\ D-40593 Düsseldorf, Germany \\ e-mail: brunoth@web.de
}

